\title{
DOPPLER DE ARTERIA UMBILICAL CON FLUJO REVERSO INTERMITENTE EN EMBARAZO GEMELAR MONOCORIAL BIAMNIÓTICO CON RCIU SELECTIVO: REPORTE DE UN CASO Y REVISIÓN DE LA LITERATURA
}

\section{A case report and review of the literature regarding umbilical artery Doppler with intermittent reversed flow in monochorionic biamniotic twin pregnancy} with selective IUGR

María L. Gálvez, M.D.*, Nadiezdha Rodríguez, M.D.**, Iván Gómez, M.D.**, Giuliana Puccini, M.D.**, Andrés Sarmiento, M.D.***, Fabio Quijano, M.D.****

Recibido: enero 29/08 - Aceptado: junio 20/08

\section{RESUMEN}

Objetivo: revisar la literatura existente acerca del retardo de crecimiento intrauterino discordante en el embarazo gemelar, en cuanto a las características de doppler de la arteria umbilical, la fisiopatología del patrón que representan sus ondas y las implicaciones como factor pronóstico de bienestar fetal y manejo.

Se presenta un caso clínico de paciente con estudio de doppler reverso intermitente de arteria umbilical en feto con restricción de crecimiento intrauterino (RCIU) discordante de gestación gemelar monocorial biamniótico.

Discusión: la literatura revisada mostró que el embarazo gemelar monocorial tiene formas úni-

\footnotetext{
* $\quad$ Residente de Ginecología y Obstetricia, Universidad El Bosque. Bogotá, Colombia. Correo electrónico: maele70@gmail.com

** Ginecoobstetra de la Unidad de Medicina Materno Fetal (UMMF). Departamento de ginecología y obstetricia hospital universitario Fundación Santa Fe de Bogotá

*** Jefe del Departamento de ginecología y obstetricia hospital universitario Fundación Santa Fe de Bogotá

**** Jefe de UMMF. Departamento de ginecología y obstetricia hospital universitario Fundación Santa Fe de Bogotá
}

cas de relaciones vasculares entre sus fetos, que hacen que se dibujen ondas doppler específicas para la arteria umbilical que tienen al parecer una correlación clínica directa con el desenlace de estos embarazos; sin embargo, falta evidencia suficiente que soporte estos hallazgos para poder, de manera concreta, definir patrones de doppler de la arteria umbilical y dar un pronóstico certero de cada uno.

Palabras clave: restricción de crecimiento intrauterino selectivo, RCIUS, embarazo gemelar monocorial biamniótico, anastomosis arterioarteriales (AAA), doppler de arteria umbilical.

\section{SUMMARY}

Objective: this article presents a review of the existing literature on selective intrauterine growth restriction (sIUGR) in monochorionic biamniotic twin pregnancies, umbilical artery Doppler's characteristics, the pathophysiology of its typical wave forms and its implications as a prognostic factor of foetal well-being and management. A case 
of intermittently reversed Doppler of the umbilical artery is presented in a monochorionic biamniotic twin pregnancy discordant growth restricted foetus.

Discussion: the literature review revealed that monochorionic pregnancies have different vascular connections between the foetuses, thereby making umbilical artery Doppler waves reveal a clear representation of communication patterns. There is an important correlation between Doppler wave type, clinical evolution and pregnancy outcome; however, insufficient evidence was produced here to support such findings and establish definitive umbilical artery Doppler patterns as a prognostic factor.

Key words: selective intrauterine growth restriction, sIUGR, monochorionic biamniotic twin pregnancy, arterioarterial anastomoses (AAA), umbilical artery Doppler.

\section{INTRODUCCIÓN}

Durante la última década, la proporción de embarazos gemelares se ha incrementado debido probablemente al uso de técnicas de reproducción asistida, siendo hoy en día un desafío mayor para el obstetra. La restricción de crecimiento intrauterino (RCIU) constituye una de las más frecuentes complicaciones del embarazo gemelar y se asocia a un incremento del riesgo de morbilidad y mortalidad perinatal. Entre un 12 y un 25\% de estos embarazos se ven afectados por alteraciones hemodinámicas de tipo RCIU selectivo, generalmente son debidas a modificaciones de la angioarquitectura de la placenta. ${ }^{1} \mathrm{No}$ hay criterios diagnósticos claros para el RCIU en los gemelos monocoriales; sin embargo, las características del flujo doppler color de la arteria umbilical se ven fuertemente influenciadas por la existencia de interconexiones vasculares, especialmente anastomosis arterioarteriales (AAA), y su comportamiento es exclusivo en este tipo de gestaciones, lo que la hace relevante en el estudio de la RCIU de embarazos gemelares monocoriales. ${ }^{2}$

El doppler color de la arteria umbilical, podría ser útil para el diagnóstico del RCIU selectivo ya que brinda una clara representación del patrón de las comunicaciones; puede mostrar tres patrones de onda doppler como son: tipo I onda positiva, tipo II onda persistentemente ausente o persistentemente reversa y tipo III onda intermitentemente reversa o intermitentemente ausente. ${ }^{2}$

Existe una correlación entre el tipo de onda doppler de la arteria umbilical con la evolución clínica y el desenlace del embarazo. ${ }^{3}$ Los avances en la tecnología han permitido una mayor comprensión de esta patología hasta el punto de permitir un mejor conocimiento de la placenta, su anatomía y la posible localización de las alteraciones vasculares. A pesar de esto, sigue siendo indispensable la correlación clínica que haga el obstetra de cada caso en el momento de decidir una conducta como desembarazar o continuar la gestación ya que involucra la vida y posiblemente el estado neurológico futuro de estos individuos.

El objetivo de este artículo es presentar el caso de una paciente con doppler reverso intermitente de arteria umbilical en feto con restricción de crecimiento intrauterino (RCIU) selectivo de gestación gemelar monocorial biamniótico y revisión de la literatura, exponiendo las características del doppler de la arteria umbilical en el contexto del RCIU selectivo, la fisiopatología del patrón que representan sus ondas, las implicaciones como factor pronóstico de bienestar fetal y la importancia de su identificación como posible determinante para estimar el momento de desembarazar.

\section{METODOLOGÍA}

Basados en el interrogante del manejo y seguimiento de embarazos gemelares monocoriales con restricción de crecimiento intrauterino y el doppler de la arteria umbilical como posible patrón paraclínico, se realizó una búsqueda de la literatura en internet en los buscadores Pubmed y Medline con los términos "Discordant growth in twins", "monochorionic twin pregnancy", "intrauterine growth restriction" y "umbilical Doppler in monochorionic twin pregnancies". 


\section{REPORTE DE CASO}

Paciente de 35 años G2P1A0V1 con embarazo gemelar monocorial biamniótico, de 28 semanas, remitida al servicio de urgencias del departamento de ginecología y obstetricia del hospital universitario Fundación Santa Fe de Bogotá, Colombia, por presentar discordancia de peso entre los fetos con sospecha de transfusión feto-fetal. Embarazo controlado con seguimiento ecográfico que evidenciaba en las últimas dos ecografías una diferencia en la edad gestacional de dos semanas, una discordancia del 30\% del peso estimado por biometría fetal, con doppler fetoplacentario inicialmente reportado normal. Presenta una segunda ecografía doppler que reporta ausencia de flujo en diástole en arteria umbilical en feto número 2. Considerando los hallazgos ecográficos se decide hospitalizar para realizar estudios complementarios por sospecha de síndrome de transfusión gemelo-gemelo.

Se realiza ecografía obstétrica y doppler fetoplacentario con equipo Voluson pro 730 General Electric con transductor volumétrico de $3,5 \mathrm{MHz}$ por parte del equipo de especialistas de la unidad de medicina materno fetal encontrando un embarazo gemelar monocorial biamniótico con biometría fetal del gemelo 1 para 27 semanas 2 días, peso estimado fetal $1.167 \mathrm{~g}$ y para el gemelo 2 una biometría de 25 semanas 3 días con un peso estimado fetal de $761 \mathrm{~g}$. Líquido amniótico con bolsillo máximo de 4,8 cm para gemelo 1 y de 4,0 cm para gemelo 2 considerados normales y no compatibles con secuencia oligohidramnios polihidramnios, presencia de vejiga en ambos gemelos por lo que se descarta síndrome de transfusión fetofetal; con una discordancia de peso fetal del 30\%. Doppler fetoplacentario para gemelo 1 normal, sin signos de redistribución hemodinámica como mecanismo compensatorio a hipoxia fetal. Doppler fetoplacentario para feto 2 de arteria umbilical persistentemente reverso durante la diástole ventricular; (figura 1) morfología del ductus venoso normal, onda atrial positiva, para los dos fetos. Se evidencia inserción marginal del cordón umbilical del gemelo dos, con territorio placentario comparativamente menor. Figura 2

Figura 1. Doppler de arteria umbilical que muestra flujo persistentemente reverso en diástole ventricular para el gemelo 2.

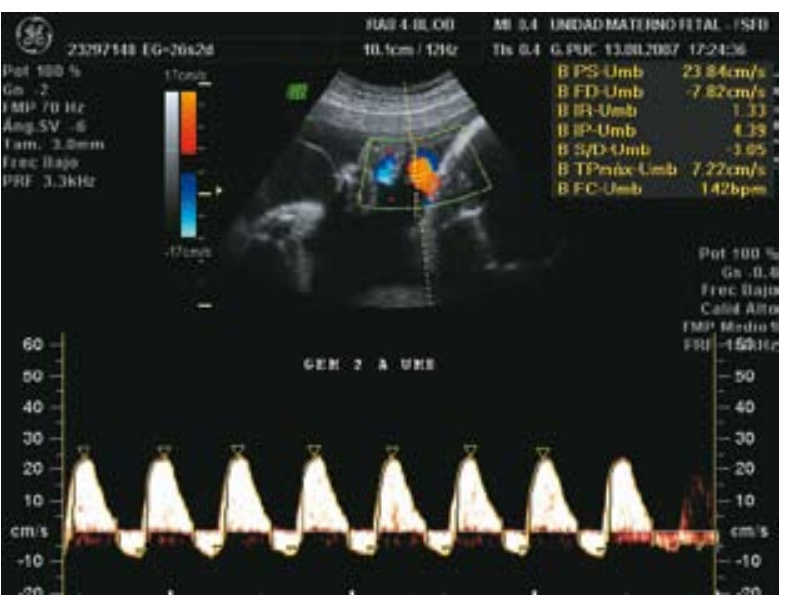

Figura 2. Ecografía obstétrica donde se evidencia inserción marginal del cordón umbilical del gemelo 2.

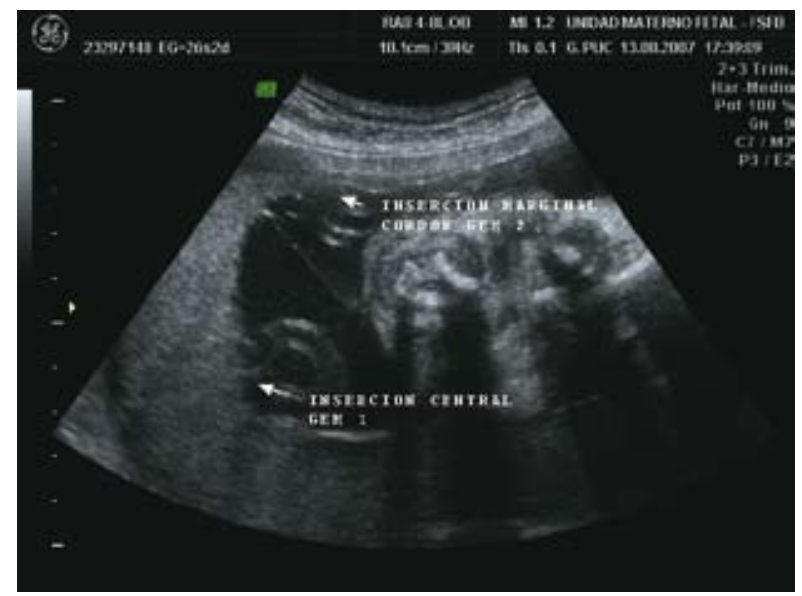

Se diagnostica restricción de crecimiento intrauterino selectivo (RCIUS) para el gemelo 2, doppler tipo V con alteración hemodinámica grave. Se decide mantener en hospitalización para monitorización diaria de bienestar fetal, con doppler fetoplacentario, monitoría fetal (NST) diaria y control de crecimiento fetal ecográfico. Se realiza un segundo doppler de la arteria umbilical que muestra un patrón de flujo intermitente reverso en el gemelo 2. Figuras 3 y 4 
Figura 3. Doppler fetoplacentario umbilical con patrón intermitente reverso. Nótese el patrón oscilatorio de las ondas.

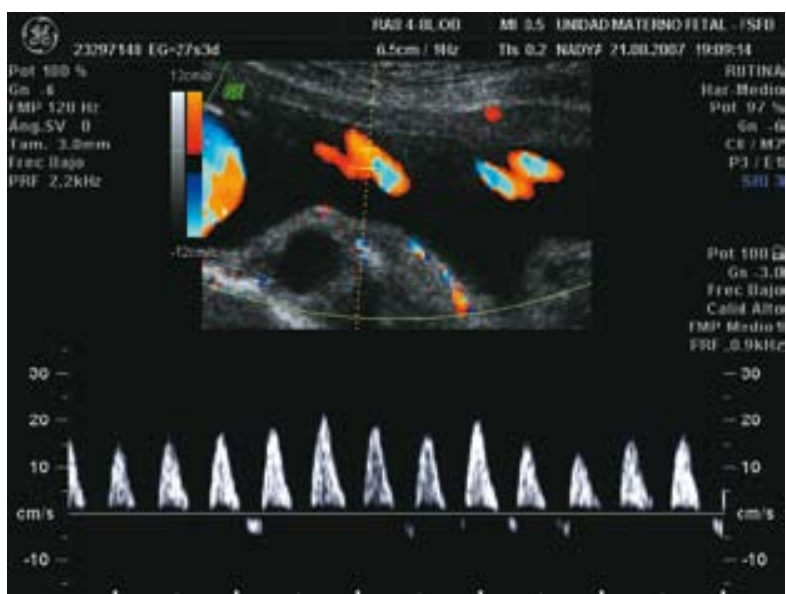

Figura 4. Patrón de flujo intermitente reverso durante la diástole en la arteria umbilical.

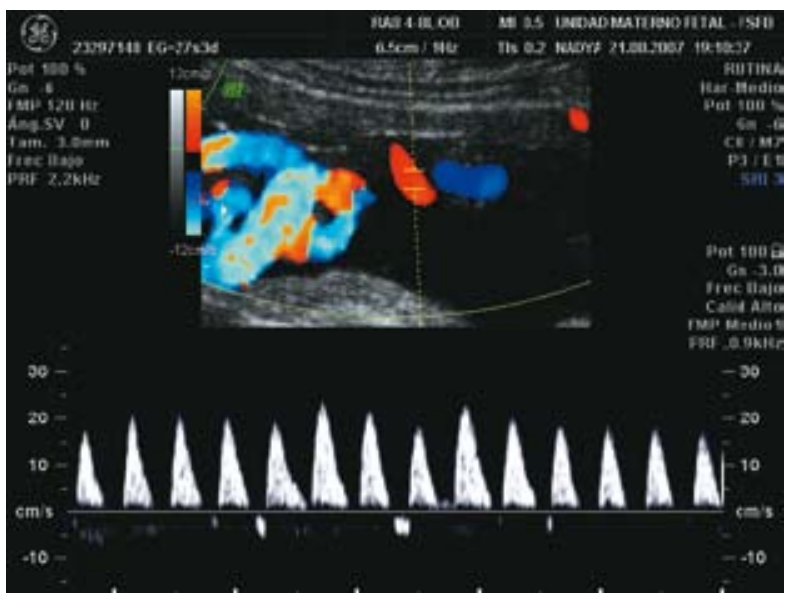

Teniendo en cuenta la dificultad del manejo en esta patología por el alto riesgo de muerte fetal inútero y la poca posibilidad de predecir un desenlace, se inicia manejo interdisciplinario con la unidad de cuidado neonatal (UCIN) y se explicó a los padres la condición de la gestación, posibles riesgos y la conducta a seguir, decisión de la cual fueron partícipes.

El seguimiento intrahospitalario permitió hacer una valoración más exhaustiva, facilitando la identificación ecográfica de una de las anastomosis vasculares de la placenta. Figuras 5 y 6 Igualmente se valora el Doppler de la arteria um- bilical y se encuentra en el gemelo 2 un patrón ondulante que explica el flujo bidireccional.

Figura 5. Anastomosis vascular superficial de los territorios placentarios

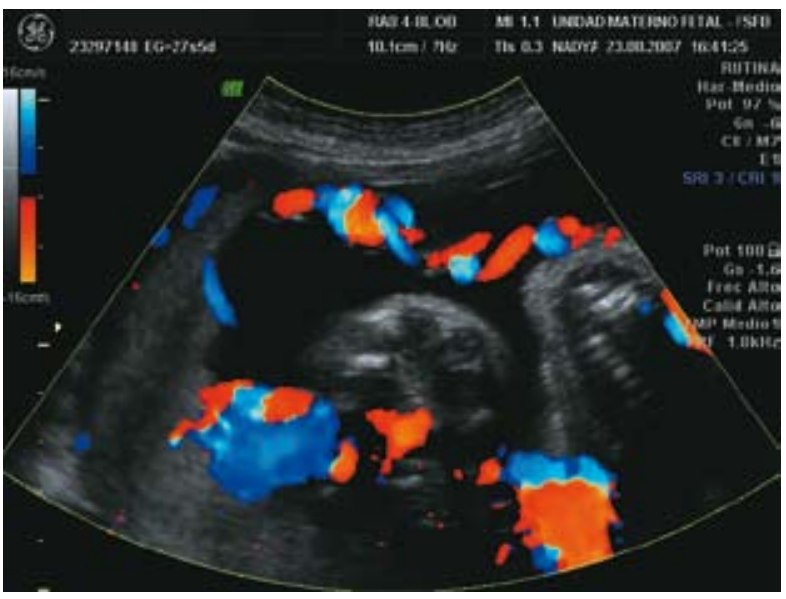

Figura 6. Doppler fetoplacentario de comunicación vascular de los territorios placentarios

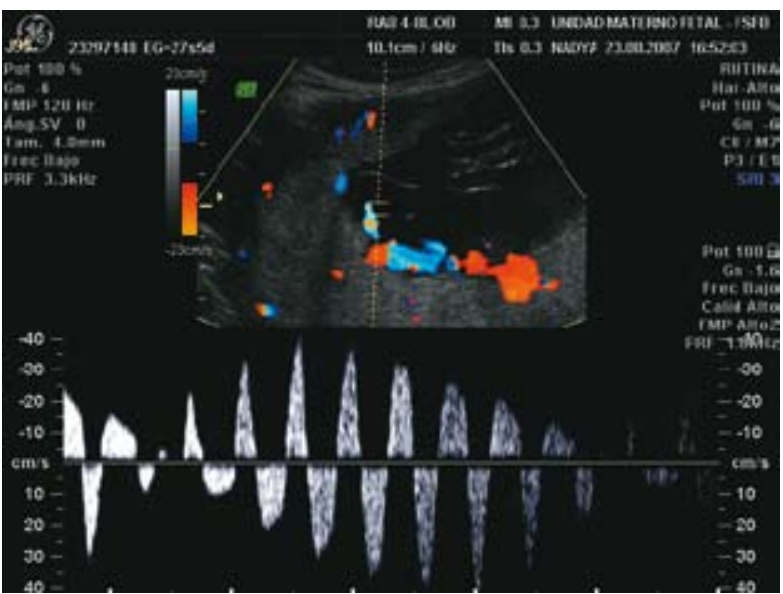

El día 16 de hospitalización se encuentra en ecografía una curva de crecimiento estancada para el gemelo 2 con un PEF de 890 g; igualmente el doppler de la arteria umbilical persiste con flujo ausente e intermitente reverso durante la diástole y se decide desembarazar, se realiza cesárea segmentaria transperitoneal sin complicaciones (en el día 17 de hospitalización) y se obtienen: recién nacido 1 masculino con peso $1.475 \mathrm{~g}$ talla $40 \mathrm{~cm}$ Ballard para 30 semanas. Gemelo 2 masculino de $865 \mathrm{~g}$ de peso. Los recién nacidos son trasladados a la UCIN para valoración exhaustiva y manejo. 
El servicio de patología informó placenta única de $698 \mathrm{~g}$ de peso, vasos tortuosos ingurgitados en la cara fetal con presencia de dos cordones umbilicales, el primero de inserción paracentral con diámetro transversal de 1,2 cm trivascular; el segundo cordón de inserción marginal de $0,5 \mathrm{~cm}$ de diámetro transverso en su parte más ancha, trivascular y ambos territorios con zonas de maduración acelerada no homogénea. Figura 7

Figura 7. Placenta donde se evidencia posible vaso anastomótico proveniente del territorio del gemelo 2 que aparenta continuar su trayecto en el territorio placentario del gemelo 1. Nótese la diferencia aparente del grosor entre los dos cordones umbilicales y la inserción marginal del cordón de la izquierda que corresponde al gemelo 2 .

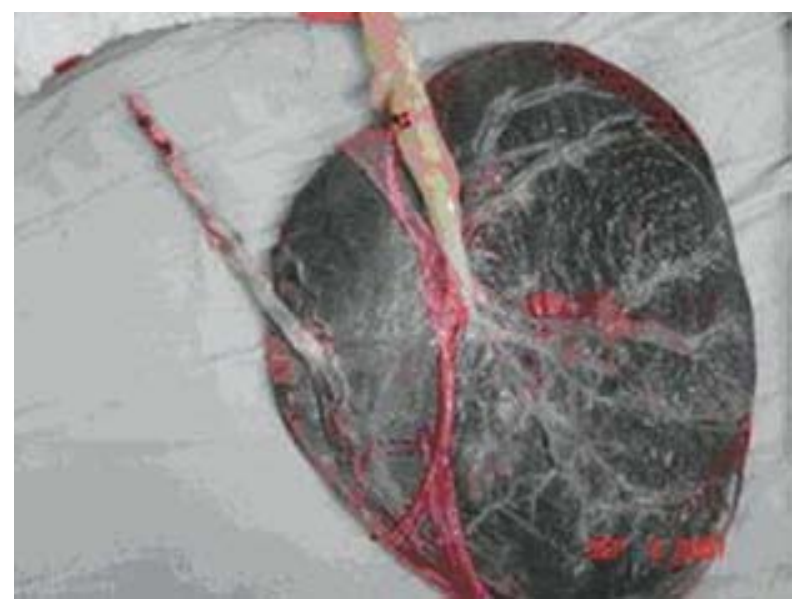

Posteriormente la paciente es trasladada a piso donde evoluciona satisfactoriamente de su posoperatorio y se da de alta de nuestra institución. Los dos recién nacidos presentaron complicaciones asociadas a la prematurez, requirieron soporte ventilatorio y manejo antibiótico hasta por un mes en la UCIN, de donde fueron trasladados a una dependencia de menor complejidad, con evolución satisfactoria.

\section{DISCUSIÓN}

El embarazo gemelar es hoy en día un desafío mayor para el ginecoobstetra; por sí mismo representa un mayor riesgo de morbimortalidad materno y perinatal. ${ }^{4}$ La incidencia de embarazo gemelar en la población es aproximadamente de 1 en 90 de todos los embarazos, de estos $30 \%$ son monocigotos idénticos y $70 \%$ son dicigotos fraternos. ${ }^{1}$

Dentro de las diferentes posibilidades del embarazo gemelar la corionicidad única es además una condición más compleja, que se encuentra en el 75\% de los gemelos que lleva consigo una mayor morbilidad comparada con los embarazos gemelares dicoriónicos. ${ }^{5,6}$

La restricción del crecimiento intrauterino selectivo en los gemelos monocoriónicos no es un proceso fácilmente explicable desde el punto de vista genético o cromosómico, ya que por definición ambos gemelos llevan los mismos genes. ${ }^{7}$ Existen tres factores importantes que se han considerado como la mayor influencia para la restricción del crecimiento intrauterino selectivo (RCIUS) en los gemelos monocoriónicos: la proporción de placenta compartida, la calidad de la implantación de cada una de las porciones de placenta y la angioarquitectura, estos tres factores determinan la magnitud del retorno venoso y la suplencia de oxígeno y nutrientes para cada feto. ${ }^{8}$

Si se considera que la circulación de ambos fetos está conectada por medio de la placenta y se generan fenómenos hemodinámicos tan importantes y variados como el síndrome de transfusión feto fetal (STFF) y la restricción de crecimiento intrauterino selectivo. ${ }^{9,10}$

Existen comunicaciones vasculares anatómicas entre las dos circulaciones, llamadas anastomosis, que pueden presentarse hasta en un $90 \%$ de estos embarazos, y pueden ser de tipo arterioarterial, (AA) arteriovenoso (AV-VA) y venovenosos (VV). ${ }^{11}$ Las comunicaciones AA y VV son típicamente superficiales y bidireccionales, mientras que las comunicaciones AV son unidireccionales de predominio en capilares profundos dentro de los cotiledones. ${ }^{12}$ El $15 \%$ de estas alteraciones tiene asociación con el síndrome de transfusión feto fetal, ${ }^{13}$ pero es importante anotar que no todas las comunicaciones vasculares de este tipo terminan desencadenando STFF. ${ }^{14}$ 
Por otra parte la restricción de crecimiento intrauterino constituye una de las más frecuentes complicaciones del embarazo gemelar y se asocia a un incremento en el riesgo de morbilidad y mortalidad perinatal, ${ }^{15}$ además de las consecuencias y efectos adversos sobre el desarrollo neurológico a largo plazo que se extienden incluso hasta la vida adulta. ${ }^{16}$

Entre un 12 y un 25\% de los embarazos monocoriales se ven afectados por alteraciones hemodinámicas de tipo RCIU selectivo, lo que quiere decir que solo uno de los fetos presenta la restricción, mientras que el otro feto tiene un crecimiento acorde con la edad gestacional. ${ }^{17}$

Para diagnosticar la RCIU en los embarazos gemelares y específicamente cuando este es selectivo, no están establecidos criterios de manera universal, algunos autores toman la circunferencia abdominal, el peso estimado fetal y el grado de discordancia entre los fetos como criterios de diagnóstico, ${ }^{1}$ específicamente estiman un crecimiento fetal discordante cuando uno de los fetos tiene un peso estimado por debajo del percentil 10 y cuando hay una diferencia de peso de uno de los fetos del 25\% con respecto al feto mayor.

Las alteraciones hemodinámicas por las anastomosis pueden ser consecuencia de una invasión trofoblástica alterada, con aumento en la resistencia vascular en la arteria uterina, y de las arterias espiraladas o por una vasoconstricción de los vasos placentarios, que sugieren una invasión trofoblástica desigual en la porción placentaria que corresponde al feto con restricción. ${ }^{12,18}$

Para valorar la RCIU selectiva se estudia el doppler color de la arteria umbilical, que muestra una clara representación del patrón de las comunicaciones, y se ha correlacionado con las distintas formas clínicas en fetos monocoriónicos con posible diagnóstico de RCIU selectivo. La arteria umbilical puede mostrar tres patrones de onda doppler como son: tipo I onda positiva, tipo II onda persistentemente ausente o persistentemente reversa al final de la diástole y tipo III onda intermitentemente reversa o intermitentemente ausente al final de la diástole; que corresponden a las anastomosis vasculares, a la dirección y a la magnitud del flujo intercambiable. ${ }^{1,19}$ Representan la colisión de dos flujos opuestos de sangre con una onda bidireccional probablemente como resultado del ritmo cardiaco asincrónico entre los gemelos. ${ }^{8}$

Las características hemodinámicas del flujo de la arteria umbilical con apariencia reversa intermitente o ausente fueron descritas para embarazos monocoriales hace ya más de veinte años, aunque hasta hace poco tiempo relativamente se han descrito asociaciones clínicas al respecto. Este fenómeno se define como la presencia de cambios cíclicos en el componente diastólico del flujo de la arteria umbilical (AU) en la imagen doppler de manera intermitente, que corresponde como ya se mencionó a la presencia invariable de anastomosis arterioarteriales (AAA) largas, que además se asocian con una evolución clínica atípica del embarazo; ${ }^{8,20}$ con un significado y una progresión distinta comparada con otras gestaciones, han sugerido un pobre resultado perinatal para los fetos que los presentan. ${ }^{20}$ Han mostrado una elevación mayor en el riesgo de muerte intrauterina para el feto pequeño en el 15\% de los casos comparado con los otros patrones (2,3\% con patrón I y 0\% para doppler con patrón II). Además tienen un riesgo mayor de deterioro del parénquima cerebral para el feto grande, inclusive si el feto pequeño sobrevive ${ }^{3}$ mientras que el patrón de flujo positivo de la arteria umbilical (AU) durante la diástole se ha correlacionado con un buen pronóstico, y las pacientes con doppler tipo II han mostrado un alto riesgo de deterioro fetal hasta de un $90 \%$ secundario a estados de hipoxia.

Hasta el momento las opciones planteadas para el manejo de embarazos gemelares monocoriales con restricción de crecimiento intrauterino selectivo incluyen el manejo expectante con vigilancia estricta del desarrollo y bienestar fetal; la terminación de embarazo del feto con RCIUS con alto riesgo de muerte in útero y la coagulación selectiva de los 
vasos comunicantes, sin embargo, faltan estudios que soporten estas decisiones clínicas. ${ }^{3}$

\section{CONCLUSIONES}

El embarazo gemelar monocorial tiene formas únicas de relaciones vasculares entre sus fetos que hacen que se dibujen ondas de doppler específicas para la arteria umbilical que adquieren al parecer una correlación clínica directa con el desenlace de estos embarazos y con los riesgos que a su vez representan, tanto para el feto pequeño como para su hermano.

Es necesario, a partir de la clasificación de los hallazgos del doppler de la arteria umbilical de estos fetos, realizar un seguimiento exhaustivo hemodinámico y de crecimiento fetal aunque por el momento no hay tiempos descritos que indiquen cuál va a ser el resultado perinatal para cada uno de estos embarazos a corto, mediano y largo plazo.

Los avances en la tecnología nos han permitido evolucionar en el manejo activo de esta patología hasta el punto de conocer su anatomía y la posible ubicación de la alteración vascular, pero sigue siendo indispensable la correlación clínica que haga el obstetra de cada caso en el momento de decidir una conducta de tan alto impacto, ya que compromete la vida y posiblemente el estado neurológico futuro de estos individuos.

\section{REFERENCIAS}

1. Lewi L, Van Schoubroeck D, Gratacós E, Witters I, Timmerman D, Deprest J. Monochorionic diamniotic twins: complications and management options. Curr Opin Obstet Gynecol 2003;15:177-94.

2. Gratacós E, Lewi L, Muñoz B, Acosta R, Hernández E, Martínez JM, et al. A classification system for selective intrauterine growth restriction in monochorionic pregnancies according to umbilical artery Doppler flow in the smaller twin. Ultrasound Obstet Gynecol 2007;30:28-34.

3. Gratacós E, Antolin E, Lewis L, Martínez JM, Hernandez-Andrade E, Acosta-Rojas R, et al. Monochorionic twins with selective intrauterine growth restriction and intermittent absent or reversed end- diastolic flow (type III): feasibility and perinatal outcome of fetoscopic placental laser coagulation. Ultrasound Obstet Gynecol 2008;31:669-75.

4. Graham GM 3rd, Gadippati S. Diagnosis and management of obstetrical complications unique to multiple gestations. Semin Perinatol 2005;29:282-95.

5. Salafia CM, Charles AK, Maas EM. Placental and fetal growth restriction. Clin Obstet Gynecol 2006;49:23656.

6. Ferreira I, Laureano C, Branco M, Fonseca M, Pinheiro A, Silva M, et al. Corionicidade e complicaçoes perinatais. Acta Med Port 2005;18:183-8.

7. Machin GA. Some causes of genotypic and phenotypic discordance in mozygotic twin pairs. Am J Med Genet 1996;61:216-28.

8. Denbow ML, Cox P. Taylor M, Hammal DM, Fisk NM. Placental angioarchitecture in monochorionic twin pregnancies: relationship to fetal growth, fetofetal transfusion syndrome and pregnancy outcome. Am J Obstet Gynecol 2000;182:417-26.

9. Gratacós E, Lewi L, Carreras E, Becker J, Higueras $\mathrm{T}$, Deprest $\mathrm{J}$, et al. Incidence and characteristics of umbilical artery intermittent absent and/or reversed end-diastolic flow in complicated and uncomplicated monochorionic twin pregnancies. Ultrasound Obstet Gynecol 2004;23:456-60.

10. Mari G, Roberts A, Detti L, Kovanci E, Ertug, BahadoSingh T, et al. Perinatal morbidity and mortality rates in severe twin-twin transfusion syndrome: results of the International Amnioreduction Registry. Am J Obstet Gynecol 2001;185:1048-51.

11. Fieni E, Gramellini D, Piantelli G, Verrotti C, Cavallotti D. Twin-Twin transfusión Syndrome: A review of tratment option. Acta Biomed 2004;75(Suppl 1):34-9.

12. Lewi L, Jani J, Cannie M, Robyr R, Ville Y, Hecher K, et al. Intertwin anastomoses in monochorionic placentas after fetoscopic laser coagulation for twin-to-twin transfusion syndrome: is there more than meets the eye? Am J Obstet Gynecol 2006;194:790-5.

13. Huber A, Dile W, Zikulnig L, Bregenzer T, Hackelöer B, Hecher K. Perinatal outcome in monochorionic twin pregnancies complicated by amniotic fluid discordance without severe twin-twin transfusion syndrome. Ultrasound Obstet Gynecol 2006;27: 48-52.

14. Nicolaides K, Rizzo G, Hecker K, Ximenes R. Doppler studies in twin pregnancy. Diploma in Fetal Medicine and ISUOG Educational Series. Visitado 
2007 Oct 16. Disponible en: http://www.centrus.com. br/DiplomaFMF/SeriesFMF/doppler/capituloshtml/ chapter_11htm

15. Chang EY. Timing of delivery in multiple gestation. Clin Obstet Gynecol 2004;47:237-47.

16. Maulik D. Managment of fetal growth restriction: an evidence-based approach. Clin Obstet Gynecol 2006;49:320-34.

17. Matijevic R, Ward S, Bajoria R. Non-invasive method of evaluation of trophoblast invasion of spiral arteries in monochorionic twins with discordant birthweight. Placenta 2002;23:93-9.

18. Vanderheyden TM, Fichera A, Pasquini L, Tan TYT Wee LY, Frusca T, et al. Increased latency of absent end- diastolic flor in the umbilical artery of monochorionic twin fetuses. Ultrasound Obstet Gynecol 2005;26: 44-9.

19. Wee LY, Taylor MJ, Vanderheyden T, Talbert D, Fisk NM. Transmitted arterio-arterial anastomosis waveforms causing cyclically intermittent absent/ reversed end-diastolic umbilical artery flow in monoochorionic twins. Placenta 2003;24:772-8.

20. Gratacós E, Carreras E, Becker J, Lewi L, Enríquez G, Perapoch J, et al. Prevalence of neurological damage in monochorionic twins with selective intrauterine growth restriction and intermittent absent or reversed end-diastolic umbilical artery flow. Ultrasound Obstet Gynecol 2004;24:159-63. 